\begin{tabular}{lr} 
DE $\begin{array}{lr}\text { DE GRUYTER } \\
\text { OPEN }\end{array}$ & $\begin{array}{r}\text { Communications in Applied } \\
\text { and Industrial Mathematics } \\
\text { ISSN 2038-0909 }\end{array}$ \\
Research Article \\
\hline Commun. Appl. Ind. Math. 7 (1), 2016, 48-67 & DOI: 10.1515/caim-2016-0018
\end{tabular}

\title{
Dynamics of a bubble rising in gravitational field
}

\author{
Enrico De Bernardis ${ }^{1 *}$, Giorgio Riccardi ${ }^{2,1}$ \\ ${ }^{1}$ CNR-INSEAN, Marine Technology Research Institute, Italy \\ ${ }^{2}$ Department of Industrial and Information Engineering, \\ Second University of Naples, Italy \\ *Email address for correspondence: enrico.debernardis@cnr.it \\ Communicated by Roberto Natalini \\ Received on November 11, 2014. Accepted on June 22, 2015.
}

\begin{abstract}
The rising motion in free space of a pulsating spherical bubble of gas and vapour driven by the gravitational force, in an isochoric, inviscid liquid is investigated. The liquid is at rest at the initial time, so that the subsequent flow is irrotational. For this reason, the velocity field due to the bubble motion is described by means of a potential, which is represented through an expansion based on Legendre polynomials. A system of two coupled, ordinary and nonlinear differential equations is derived for the vertical position of the bubble center of mass and for its radius. This latter equation is a modified form of the Rayleigh-Plesset equation, including a term proportional to the kinetic energy associated to the translational motion of the bubble.
\end{abstract}

Keywords: cavitation, bubble dynamics, Rayleigh-Plesset equation.

AMS subject classification: 76B45, 35Q35, 35R37.

\section{Introduction}

The phenomenon of cavitation is, in many cases, a crucial aspect for the hydrodynamic behaviour of the propulsion system of a ship, as well as for the noise radiated. Along with a large effort in the development of CFD codes aimed at improving the capability of simulating multiphase flows, significant work is devoted by the fluid dynamics community to achieve more insight in cavitation through accurate modeling of the relevant bubble dynamics [1]. A number of papers, over the last decades, investigated several aspects of the evolution of gas (and vapour) bubbles moving within a steady or flowing liquid. Here we are particularly interested here to those works contributing to a better understanding of the interaction between the translating motion of a bubble and the characteristics of its shape and size changing in time. Two main groups of works can be distinguished in this respect. 


\section{Dynamics of a bubble rising in gravitational field}

The first group deals with the coupled dynamics of a bubble moving in an acoustic field. It is well represented by papers of Reddy and Szeri $[2,3]$, and Doinikov [4-6]. The radial motion of the bubble is determined through the Rayleigh-Plesset equation [1], with corrections to take into account the compressibility of liquid, as the effects of a forcing acoustic pressure field. This is represented in the equation for the translation, where the ratio of density of fluid inside and outside the bubble does not play any role, while other dynamical components such as drag and, except in [5], Bjerknes forces [7], are taken into account. Shaw [8] considers interactions between axisymmetric shape distortions, axial translational motion, and volume oscillations of a gas bubble in an inviscid, incompressible liquid, and represents the surface deformation using a complete set of Legendre polynomials, assuming that both this deformation and the translational motion are small.

In the second group, the interaction within shape oscillation and translating motion of a bubble rising due to gravitational force is studied. Yang et al. [9] deal with two problems where the rectilinear rise of a bubble undergoes a transient behavior. The evolution of bubble shape from release to steady state is simulated numerically by an incompressible axisymmetric Navier-Stokes solver; while the rising motion of a bubble, which expands or contracts due to a change in the ambient pressure, is studied analytically with no account of the density ratio (that is, by neglecting bubble mass compared to added mass). Klaseboer and Khoo [10] introduce an equivalent radius and an equivalent wall velocity for the study of non-spherical oscillations of the bubble. A modified form of Rayleigh-Plesset equation is solved using a boundary integral method. The effect of gravity on the shape evolution of the bubble is investigated, but in this case the translation dynamics is not represented explicitly. Amaya-Bower and Lee [11] use a Lattice Boltzmann Method. Terminal shape and Reynolds number depend on size of bubble, surface tension, viscosity, and density of surrounding fluid (in good agreement with results obtained by Rodrigue [12] through correlations). From a qualitative point of view this scheme does not allow direct comparisons with our model; on the other hand, the results reveal the role played by the density ratio, which is the main improvement of the present work, compared to those mentioned here that are based on the same modeling. Gordillo et al. [13] obtain a theoretical model for a rising and pulsating bubble with shape deformation, similar to that of Doinikov [5]. Results for the coupled bubble motion at low Reynolds number are compared with numerical predictions from a Navier-Stokes solver. Finally, in the work of Tuteja et al. [14] the motion of a spherically pulsating gas bubble under gravity is studied as the center of mass moves in a vertical plane. As it 


\section{E. De Bernardis, G. Riccardi}

is seen for several examples above, a system of coupled nonlinear ordinary differential equation is obtained, which describes the interaction between the radial and the translation dynamics of the bubble. In this case the Lagrange formalism is used to derive the equations of motion, again neglecting the mass of the bubble in the comparison with the mass of the displaced liquid. It is the the most similar to the work presented here: when the initial value of the horizontal component of the velocity of the center of mass is set to zero, the model reduces to one representing the vertical motion of the bubble coupled with the spherical pulsation of its surface. The study is focused on the effects of changing gravity, but the results exhibits, from a qualitative point of view, strong similarity in the resulting structure of interacting motions.

The behaviour of a spherical gas bubble rising in a liquid is studied in the present paper. The simple model derived here is intended to be the starting point for a more comprehensive theoretical tool aimed at interpreting and predicting the behaviour of cavitation bubbles on hydrodynamics devices. So, the comparison of with existing studies on the subject, as it is seen from those mentioned above, suffers from inhomogeneity of methods and objectives. However, most models are, in some respects, more refined than that proposed here, but have to pay a tribute to being fitted for particular situations, in terms of force field or kind of motion. In the present work the attempt is made to build, through subsequent upgrades, a model for the general dynamics of a bubble, with the accuracy required by a reliable representation of subsequent physical effects such as, for example, sound generation.

The paper is organized in the following way. In section 2 , the general scheme is given, and the derivation of equations of motion for the spherical bubble is prepared by writing the form of the absolute velocity potential for a pulsating and translating bubble. In section 3, the equation for the one-dimensional rising motion of the bubble center of mass is derived. It extends the general formula for the vertical motion of a rigid sphere subject to gravity force by taking into account the effect of radial pulsation of the bubble surface. A modified form of the Rayleigh-Plesset equation is obtained in section 4, describing the oscillation of the bubble radius: it includes a term that is proportional to the kinetic energy associated with the translational motion of the center of mass. Some qualitative aspects of numerical results are presented in section 5. An outline of future work for further development of the model is given in section 6 . 


\section{Dynamics of a bubble rising in gravitational field}

\section{Flowfield about the bubble}

In this section, the velocity field in the liquid about a spherical bubble is investigated. A bubble of vapour/gas is surrounded by an isochoric, inviscid liquid. The shape of the bubble is assumed spherical at any time, with a time-dependent radius $R$. A system of cartesian coordinates is used, having a vertical $z$-axis - the gravity acceleration has components $(0,0,-g)$, with $g>0$ - passing through the initial position of the center of mass of the bubble $\boldsymbol{x}_{\mathrm{cm}}(0)$. At the initial time, the liquid is at rest, while the center of mass of the bubble and/or its spherical surface move at prescribed velocities $\dot{\boldsymbol{x}}_{\mathrm{cm}}$ (along the $z$-direction) and $\dot{R}$, respectively.

The general form of the velocity potential in the liquid is given by:

$$
\varphi(r, \mu ; t)=\sum_{k=0}^{\infty} c_{k}(t) \frac{P_{k}(\mu)}{r^{k+1}}+\sum_{m=1}^{\infty} d_{m}(t) P_{m}(\mu) r^{m}, \quad t \geq 0, \quad r>R,
$$

where $r$ is the distance from the bubble center, $\mu$ is the cosine of co-latitude angle $\theta(\theta=0$ along the positive $z$ semi-axis $)$ and $P_{j}$ is the $j$-th Legendre polynomial. The condition of vanishing velocity potential far from the bubble leads to $d_{m}=0$ for $m=1,2, \ldots$, while the Neumann boundary condition at the bubble surface $(r=R)$

$$
\left.u_{r}\right|_{r=R}=\left.\frac{\partial \varphi}{\partial r}\right|_{r=R}=-c_{0} \frac{P_{0}(\mu)}{R^{2}}-2 c_{1} \frac{P_{1}(\mu)}{R^{3}}=\dot{R}(t) P_{0}(\mu)+\dot{z}_{\mathrm{cm}} P_{1}(\mu),
$$

gives

$$
c_{0}=-R^{2} \dot{R}, \quad c_{1}=-\frac{1}{2} R^{3} \dot{z}_{\mathrm{cm}}, \quad c_{k}=0 \text { for } m \geq 2 .
$$

In order to apply Bernoulli theorem, the time derivative of potential (1), in which only $c_{0}$ and $c_{1}$ are nonzero, has to be evaluated. Before performing this calculation, it must be considered that $\varphi(1)$ is written in a spherical coordinate system having its origin at the center of the bubble and that this point moves in time. For this reason, it is worthwhile to rewrite $\varphi$ in cartesian coordinates

$$
\varphi=\frac{c_{0}}{\left[x^{2}+y^{2}+\left(z-z_{\mathrm{cm}}\right)^{2}\right]^{1 / 2}}+c_{1} \frac{z-z_{\mathrm{cm}}}{\left[x^{2}+y^{2}+\left(z-z_{\mathrm{cm}}\right)^{2}\right]^{3 / 2}}
$$

and, then, to take the time derivative (by keeping $x, y, z$ fixed)

$$
\frac{\partial \varphi}{\partial t}=\frac{\dot{c}_{0}}{r} P_{0}+\frac{c_{0} \dot{z}_{\mathrm{cm}}+\dot{c}_{1}}{r^{2}} P_{1}+\frac{2 c_{1} \dot{z}_{\mathrm{cm}}}{r^{3}} P_{2} .
$$




\section{E. De Bernardis, G. Riccardi}

By using the Legendre coefficients (2), the above time derivative, the radial and tangential components of the velocity and the kinetic energy per unit mass are written as functions of $R, \dot{R}$ and $\dot{z}_{\mathrm{cm}}$ :

$$
\begin{gathered}
\frac{\partial \varphi}{\partial t}=-\frac{R}{r}\left(2 \dot{R}^{2}+R \ddot{R}\right) P_{0}-\frac{R^{2}}{r^{2}}\left(\frac{5}{2} \dot{R} \dot{z}_{\mathrm{cm}}+\frac{1}{2} R \ddot{z}_{\mathrm{cm}}\right) P_{1}-\frac{R^{3}}{r^{3}} \dot{z}_{\mathrm{cm}}^{2} P_{2} \\
u_{r}=\frac{R^{2}}{r^{2}} \dot{R} P_{0}+\frac{R^{3}}{r^{3}} \dot{z}_{\mathrm{cm}} P_{1} \quad u_{\theta}=-\frac{1}{r} \frac{\partial \varphi}{\partial \theta}=-\frac{1}{2} \frac{R^{3}}{r^{3}} \dot{z}_{\mathrm{cm}} \sin \theta \\
\frac{|\boldsymbol{u}|^{2}}{2}=\frac{1}{4}\left(2 \frac{R^{4}}{r^{4}} \dot{R}^{2}+\frac{R^{6}}{r^{6}} \dot{z}_{\mathrm{cm}}^{2}\right) P_{0}+\frac{R^{5}}{r^{5}} \dot{R} \dot{z}_{\mathrm{cm}} P_{1}+\frac{1}{4} \frac{R^{6}}{r^{6}} \dot{z}_{\mathrm{cm}}^{2} P_{2} .
\end{gathered}
$$

The equations for the dynamics of the center of mass and of the radius of the spherical bubble are now deduced, by using the time derivative of the potential (3) and the kinetic energy (5).

\section{Dynamics of the center of mass}

The position of the center of mass of the bubble is:

$$
\boldsymbol{x}_{\mathrm{cm}}=\frac{1}{m_{\mathrm{b}}} \int_{\mathscr{V}} d V \rho_{\mathrm{b}} \boldsymbol{x},
$$

where $\rho_{\mathrm{b}}$ is the density of vapour/gas inside the bubble and $m_{\mathrm{b}}$ is its (constant) mass. Hereafter, the mean value of the vapour/gas density $\bar{\rho}_{\mathrm{b}}$ on the volume $\mathscr{V}$ filled by the bubble will be also used, so that its mass $\left(m_{\mathrm{b}}\right)$ will be written as $\mathscr{V} \bar{\rho}_{\mathrm{b}}$. The second time derivative of $\boldsymbol{x}_{\mathrm{cm}}$ is

$$
\ddot{\boldsymbol{x}}_{\mathrm{cm}}=\frac{1}{m_{\mathrm{b}}} \int_{\mathscr{V}} d V \rho_{\mathrm{b}} \frac{D \boldsymbol{u}}{D t},
$$

where it has been taken into account that gas density satisfies continuity equation $D \rho_{\mathrm{b}} / D t+\rho_{\mathrm{b}} \boldsymbol{\nabla} \cdot \boldsymbol{u}=0$. On the other hand, the motion of vapour/gas inside the bubble is governed by the momentum equation $\rho_{\mathrm{b}} D \boldsymbol{u} / D t=-\nabla p_{\mathrm{b}}-\rho_{\mathrm{b}} g \boldsymbol{e}_{z}\left(\boldsymbol{e}_{z}\right.$ being the unit vector in vertical direction), so that the acceleration of the center of mass can be rewritten as:

$$
\ddot{\boldsymbol{x}}_{\mathrm{cm}}=\frac{1}{m_{\mathrm{b}}} \int_{\mathscr{V}} d V\left(-\nabla p_{\mathrm{b}}-\rho_{\mathrm{b}} g \boldsymbol{e}_{z}\right)=-\frac{1}{m_{\mathrm{b}}} \int_{\mathscr{B}} d S p_{\mathrm{b}} \boldsymbol{n}-g \boldsymbol{e}_{z},
$$

where $\mathscr{B}$ is the bubble surface. The use of Green formulae at the righthand side of the above equation is forced by the fact that the motion of 


\section{Dynamics of a bubble rising in gravitational field}

the vapour/gas inside the bubble will not be considered here and, as a consequence, the pressure inside the bubble remains unknown. The only information we have about $p_{\mathrm{b}}$ concerns its behavior on the bubble surface. Indeed, the balance of normal stresses between vapour/gas and liquid faces of this surface gives $p_{\mathrm{b}}$ in terms of the pressure $p$ in the liquid: $p_{\mathrm{b}}=p+2 \sigma / R$, $\sigma$ being the surface tension coefficient. Moreover, by using the Bernoulli law

$$
\frac{\partial \varphi}{\partial t}+\frac{|\boldsymbol{u}|^{2}}{2}+\frac{p}{\rho}+g z=\frac{p_{\infty}}{\rho}
$$

where $p+\rho g z \rightarrow p_{\infty}$ as $|\boldsymbol{x}| \rightarrow \infty$, the vapour/gas pressure $p_{\mathrm{b}}$ is related to the dynamics of the surrounding liquid

$$
p_{\mathrm{b}}=p_{\infty}-\rho g z-\rho\left(\frac{\partial \varphi}{\partial t}+\frac{|\boldsymbol{u}|^{2}}{2}\right)+\frac{2 \sigma}{R}
$$

This form of $p_{\mathrm{b}}$ is now inserted into the right-hand side of Equation (6), which results in:

$$
\ddot{\boldsymbol{x}}_{\mathrm{cm}}=g \boldsymbol{e}_{z}\left(\frac{\rho}{\bar{\rho}_{\mathrm{b}}}-1\right)+\frac{\rho}{m_{\mathrm{b}}} \int_{\mathscr{B}} d S \boldsymbol{n}\left(\frac{\partial \varphi}{\partial t}+\frac{|\boldsymbol{u}|^{2}}{2}\right) .
$$

Equation (8) is now specified in the one-dimensional case, by taking into account that

$(9)$

$$
\frac{\partial \varphi}{\partial t}+\frac{|\boldsymbol{u}|^{2}}{2}=-\left(R \ddot{R}+\frac{3}{2} \dot{R}^{2}-\frac{\dot{z}_{\mathrm{cm}}^{2}}{4}\right) P_{0}-\left(\frac{3}{2} \dot{R} \dot{z}_{\mathrm{cm}}+\frac{1}{2} R \ddot{z}_{\mathrm{cm}}\right) P_{1}-\frac{3}{4} \dot{z}_{\mathrm{cm}}^{2} P_{2},
$$

according to the forms of $\partial \varphi / \partial t(3)$ and $|\boldsymbol{u}|^{2} / 2$ (5). By using the result (9), the integral in Equation (8) is evaluated as:

$$
\begin{aligned}
\frac{\rho}{m_{\mathrm{b}}} \int_{\mathscr{B}} d S \boldsymbol{n}\left(\frac{\partial \varphi}{\partial t}+\frac{|\boldsymbol{u}|^{2}}{2}\right) & =2 \pi R^{2} \frac{\rho}{m_{\mathrm{b}}} \boldsymbol{e}_{z} \int_{-1}^{1} d \mu P_{1}\left(\frac{\partial \varphi}{\partial t}+\frac{|\boldsymbol{u}|^{2}}{2}\right) \\
& =-\boldsymbol{e}_{z} \frac{\rho}{\bar{\rho}_{\mathrm{b}}}\left(\frac{3}{2} \frac{\dot{R}}{R} \dot{z}_{\mathrm{cm}}+\frac{1}{2} \ddot{z}_{\mathrm{cm}}\right) .
\end{aligned}
$$

Once this result is inserted into Equation (8), it is easily seen that the $x$ and $y$ components of $\boldsymbol{x}_{\mathrm{cm}}$ do not change in time, so that the center of mass of the bubble moves along the $z$-axis. Moreover, the acceleration $\ddot{z}_{\mathrm{cm}}$ appears also in the right-hand side of the resulting equation of motion. The well-known concept of added mass follows just from this important remark. By naming 


\section{E. De Bernardis, G. Riccardi}

the ratio $\bar{\rho}_{\mathrm{b}} / \rho$ as $\varepsilon(\varepsilon \ll 1$ in the case of the bubble), the one-dimensional equation of motion of the center of mass is finally written as:

$$
\ddot{z}_{\mathrm{cm}}=2 g \frac{1-\varepsilon}{1+2 \varepsilon}-\frac{3}{1+2 \varepsilon} \frac{\dot{R}}{R} \dot{z}_{\mathrm{cm}} .
$$

Note that this equation reduces to the one for the dynamics of a rigid sphere (see Equation 6.8 .20 on page 454 of [15]) by enforcing $R \equiv$ constant. The dependence on $\varepsilon$ of terms on the right-hand side of Equation (10) is the main difference with respect to the corresponding Equation 15 in [14], derived by implicitly assuming $\varepsilon=0$.

\section{Dynamics of the bubble radius}

The equation for the dynamics of the bubble radius will be now derived by using Bernoulli equation (in the Appendix, the same equation is derived from the kinetic energy balance).

The vapour/gas pressure inside the bubble $p_{\mathrm{b}}$, or more precisely its mean value on the bubble surface $\bar{p}_{\mathrm{b}}$, is usually related to its volume $\mathscr{V}$ by means of an equation of state. By assuming that the motion is isothermal, it is generally accepted [1] that the product $\mathscr{V} \bar{p}_{\mathrm{b}}$ is kept constant along the motion. As a consequence, once the radius $R$ of the bubble is known, the same holds for mean vapour/gas pressure $\bar{p}_{\mathrm{b}}$, too. In the present flow, the vapour/gas pressure $p_{\mathrm{b}}$ is easily obtained by inserting the form of $\partial \varphi / \partial t+|\boldsymbol{u}|^{2} / 2$ (9) into the balance of the normal stresses across the bubble surface (7):

$$
\begin{aligned}
\frac{p_{\mathrm{b}}}{\rho}= & \left(\frac{p_{\infty}}{\rho}+\frac{2 \sigma}{\rho R}-g z_{\mathrm{cm}}+\frac{3}{2} \dot{R}^{2}+R \ddot{R}-\frac{1}{4} \dot{z}_{\mathrm{cm}}^{2}\right) P_{0} \\
& +\left(-g R+\frac{3}{2} \dot{R} \dot{z}_{\mathrm{cm}}+\frac{1}{2} R \ddot{z}_{\mathrm{cm}}\right) P_{1}+\frac{3}{4} \dot{z}_{\mathrm{cm}}^{2} P_{2},
\end{aligned}
$$

which gives the mean value of the vapour/gas pressure as:

$$
\begin{aligned}
\frac{\bar{p}_{\mathrm{b}}}{\rho} & =\frac{1}{2} \int_{-1}^{1} d \mu \frac{p_{\mathrm{b}}}{\rho} \\
& =\frac{p_{\infty}}{\rho}+\frac{2 \sigma}{\rho R}-g z_{\mathrm{cm}}+\frac{3}{2} \dot{R}^{2}+R \ddot{R}-\frac{1}{4} \dot{z}_{\mathrm{cm}}^{2} .
\end{aligned}
$$

This relation is here reread as a modified form of the well-known RayleighPlesset equation [1]:

$$
\ddot{R}=\frac{1}{R}\left(-\frac{3}{2} \dot{R}^{2}+\frac{1}{4} \dot{z}_{\mathrm{cm}}^{2}+P\right),
$$

having introduced the function $P=\left(\bar{p}_{\mathrm{b}}-p_{\infty}\right) / \rho+g z_{\mathrm{cm}}-2 \sigma /(\rho R)$ that depends on $R$ and $z_{\mathrm{cm}}$, only. 


\section{Dynamics of a bubble rising in gravitational field}

\section{Numerical results}

A total of nine sample cases are shown among the simulations conducted using the dynamical model of rising bubble based on Equations (10) and (13). In all cases the bubble is released at $1 \mathrm{~m}$ depth in the liquid and has initial radius $R_{0}=0.01 \mathrm{~m}$ in almost all the cases $\left(R_{0}\right.$ will be given if it differs from that value), selected as the length scale to define nondimensional quantities. As time scale, we take the quantity $T_{0}=2 \pi / \omega_{0}$, where $\omega_{0}$ is the natural (circular) frequency of oscillation of the bubble, given as $\omega_{N}$ by Brennen [1]. However, while the definition of $\omega_{N}$ in [1] is based on the equilibrium radius $R_{E}$ (the value of $R$ for a bubble with a given amount of gas to be in equilibrium at the given condition of external pressure), $\omega_{0}$ is calculated here using $R_{0}$, which is made the equilibrium radius at the given external pressure by properly choosing the amount of gas contained in the bubble.

The nine test cases mentioned above arise from the combination of three different initial values of radial velocity and three initial values of velocity of the center of mass.

Figure 1 shows results for a bubble starting with vertical velocity $\dot{z}_{\text {cm0 }}=0 \mathrm{~m}$. As for the other two conditions, curves for (nondimensional) $R$ (top) and $z_{\mathrm{cm}}$ (bottom) are reported, for three values of the initial radial velocity: $\dot{R}_{0}=10 \mathrm{~m} / \mathrm{s}$ (expanding bubble), $\dot{R}_{0}=0 \mathrm{~m} / \mathrm{s}$, and $\dot{R}_{0}=-10 \mathrm{~m} / \mathrm{s}$ (contracting bubble). Figure 2 shows, for the same initial conditions, curves for (nondimensional) $\dot{R}$ (top) and $\dot{z}_{\mathrm{cm}}$ (bottom).

Following the scheme above, Figures 3 and 4 show results for the same configuration of initial conditions except for the initial vertical velocity, now $\dot{z}_{\mathrm{cm} 0}=-1 \mathrm{~m} / \mathrm{s}$, while Figures 5 and 6 show results for the initial vertical velocity $\dot{z}_{\mathrm{cm} 0}=-2 \mathrm{~m} / \mathrm{s}$.

Furthermore, since curves of $R, \dot{R}$, and $\dot{z}_{\mathrm{cm}}$ for the case with zero initial value of $\dot{R}$ do not show effectively when reported along curves for other cases, we have drawn those results - and the corresponding $z_{\mathrm{cm}}$ - in a proper scale in Figures 7 and 8.

We start from the last figures to illustrate the behaviour of the system. Looking at the top Figure 7, we see that in the case the bubble is at rest at $t=0$, that is $\dot{R}_{0}=\dot{z}_{\mathrm{cm} 0}=0 \mathrm{~m} / \mathrm{s}$, the radius start expanding very slowly (without oscillations), as a consequence of the gravity acceleration, that drives the bubble upward. In fact, this results in a twofold effect of the upward velocity of the center of mass: it makes the bubble feel a decreasing external pressure, and also contributes a source term in Equation (13). As we move to the curves representing the two cases with nonzero $\dot{z}_{\text {cm0 } 0}$, we observe that this initial (negative) velocity, while pushing the bubble 


\section{E. De Bernardis, G. Riccardi}

downward, causes the radius to oscillate, with amplitude affected by the value of $\dot{z}_{\mathrm{cm} 0}$. Besides, in both cases, the mean value of this oscillation seems to follow (qualitatively) the curve of current vertical position of the bubble. A similar effect of the nonzero $\dot{z}_{\mathrm{cm} 0}$ is observed on the radial velocity, as shown in Figure 8.

We return to Figures 1, 3 and 5, to look at the trends of $R$ and $z_{\mathrm{cm}}$ for the cases of nonzero $\dot{R}_{0}$. The three tests show similar behaviour: radial oscillations (with slowly increasing mean value) of the same amplitude for $\dot{R}_{0}=10 \mathrm{~m} / \mathrm{s}$ and $\dot{R}_{0}=-10 \mathrm{~m} / \mathrm{s}$ (same $\dot{z}_{\mathrm{cm} 0}$ ), distinguished only for a phase shift. The same is true for the vertical motion of the center of mass. This is characterized, in all cases, by a jagged profile, with very steep segments caused by the spikes in the $\dot{z}_{\mathrm{cm}}$ curves, shown in Figures 2, 4 and 6. Curves of the radial velocity also follow the same pattern in the three cases, with an apparently zero mean value and a slowly time-varying amplitude, whose rate seems to be affected by the trend of velocity of the center of mass: amplitude increases at negative $\dot{z}_{\mathrm{cm}}$ and vice versa.

Finally, Figure 9 shows, with regard to the translational motion, the effect of neglecting the mass of the bubble, as it is done in most previous papers referenced here, in particular [14]. This means to enforce $\varepsilon=0$ during the numerical integration of Equation (10), rather than calculating $\varepsilon$ from the time-varying bubble volume. As a consequence, especially at the beginning of motion, peak values in the $\dot{z}_{\mathrm{cm}}$ time history are significantly overestimated, resulting in a clear departure of the $z_{\mathrm{cm}}$ curve.

\section{Concluding remarks}

A system of two coupled ordinary differential equations has been obtained to represent the vertical motion of the center of mass of a gas/vapour bubble through an inviscid and isochoric liquid and the spherical pulsation of its surface. As a main enhancement of existing formulations, the model accounts for the mass of the bubble in the equation describing its translational motion.

This model is a starting point to represent the general dynamics of a gas/vapour bubble through a liquid, so several aspects of the physical complexity of the motion are not accounted at this stage. In the near future we plan to enhance the model by including the capability to deal with: (i) effect of free surface and solid walls; (ii) non spherical oscillation of the bubble and three-dimensional translational motion of the center of mass; (iii) compressibility of liquid and thermodynamic behaviour of gas inside the bubble. By addition of these features, the model will fit the requirements for application to complex flow phenomena. 
Dynamics of a bubble rising in gravitational field
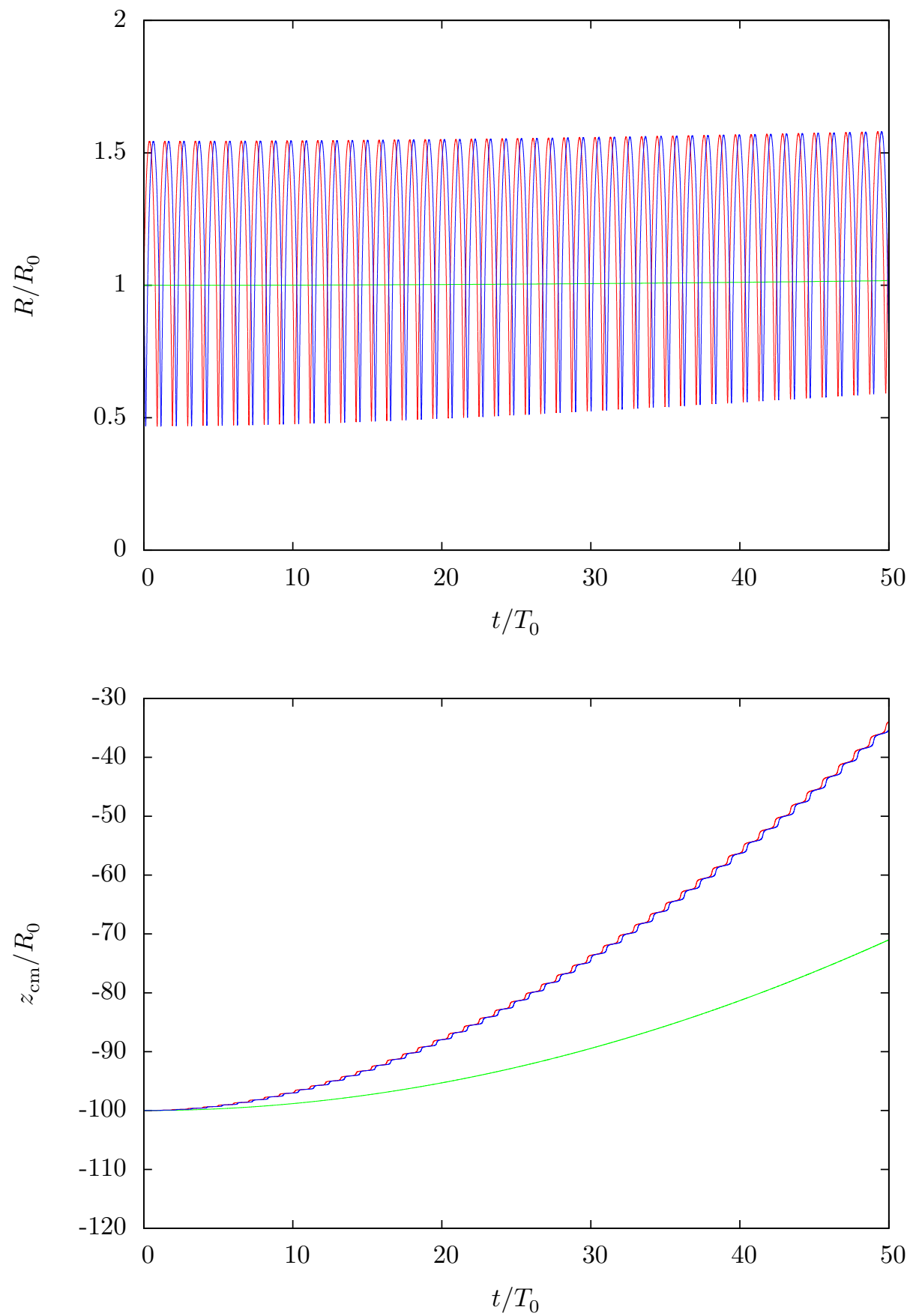

Figure 1. Bubble radius (top) and position of the center of mass (bottom). Data: $\dot{z}_{\mathrm{cm} 0}=$ $0 \mathrm{~m} / \mathrm{s}$, and $\dot{R}_{0}=10 \mathrm{~m} / \mathrm{s}$ (red), $\dot{R}_{0}=0 \mathrm{~m} / \mathrm{s}$ (green), $\dot{R}_{0}=-10 \mathrm{~m} / \mathrm{s}$ (blue). 
E. De Bernardis, G. Riccardi
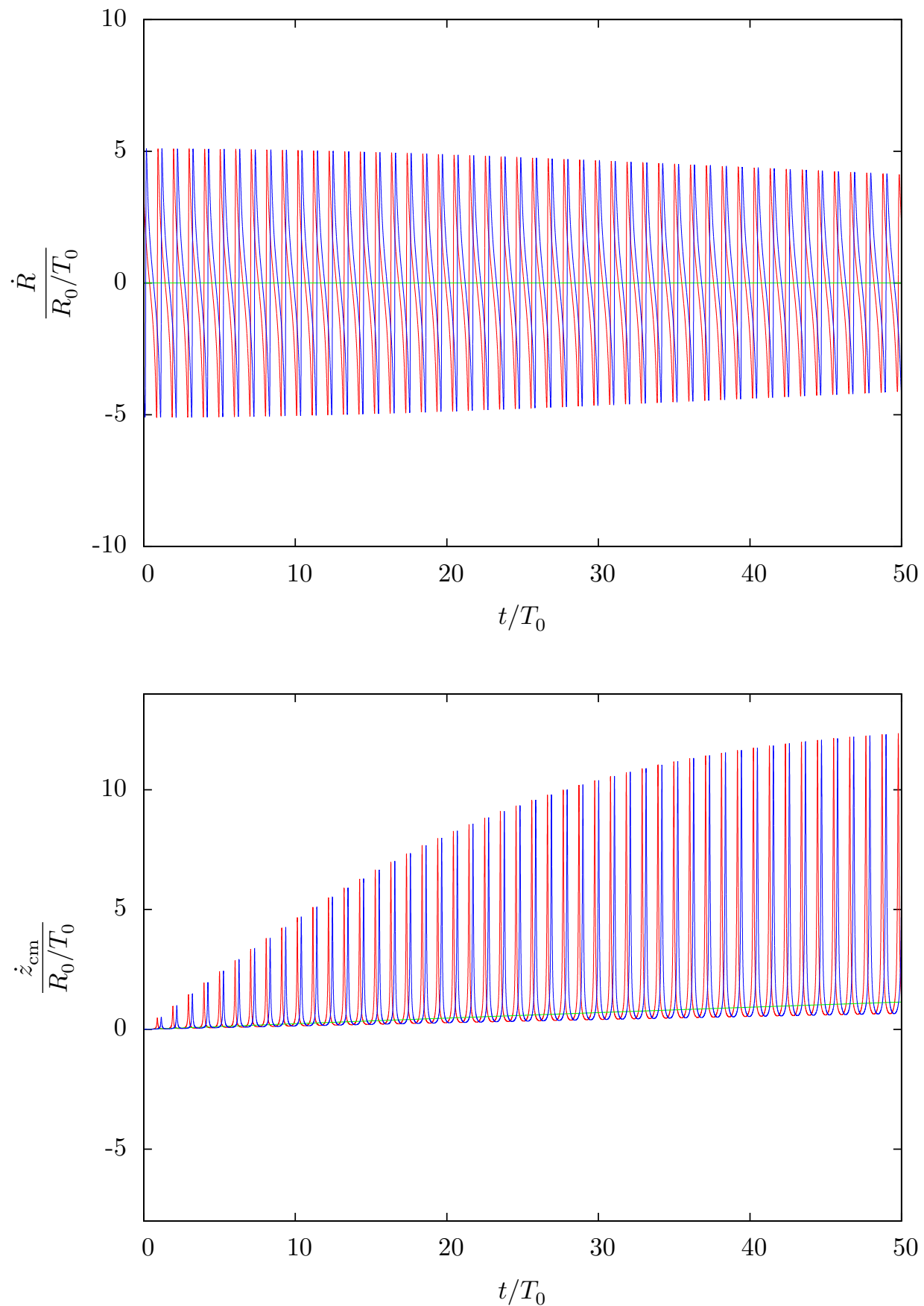

Figure 2. Radial velocity (top) and velocity of the center of mass (bottom). Data: $\dot{z}_{\mathrm{cm} 0}=0 \mathrm{~m} / \mathrm{s}$, and $\dot{R}_{0}=10 \mathrm{~m} / \mathrm{s}$ (red), $\dot{R}_{0}=0 \mathrm{~m} / \mathrm{s}$ (green), $\dot{R}_{0}=-10 \mathrm{~m} / \mathrm{s}$ (blue). 
Dynamics of a bubble rising in gravitational field
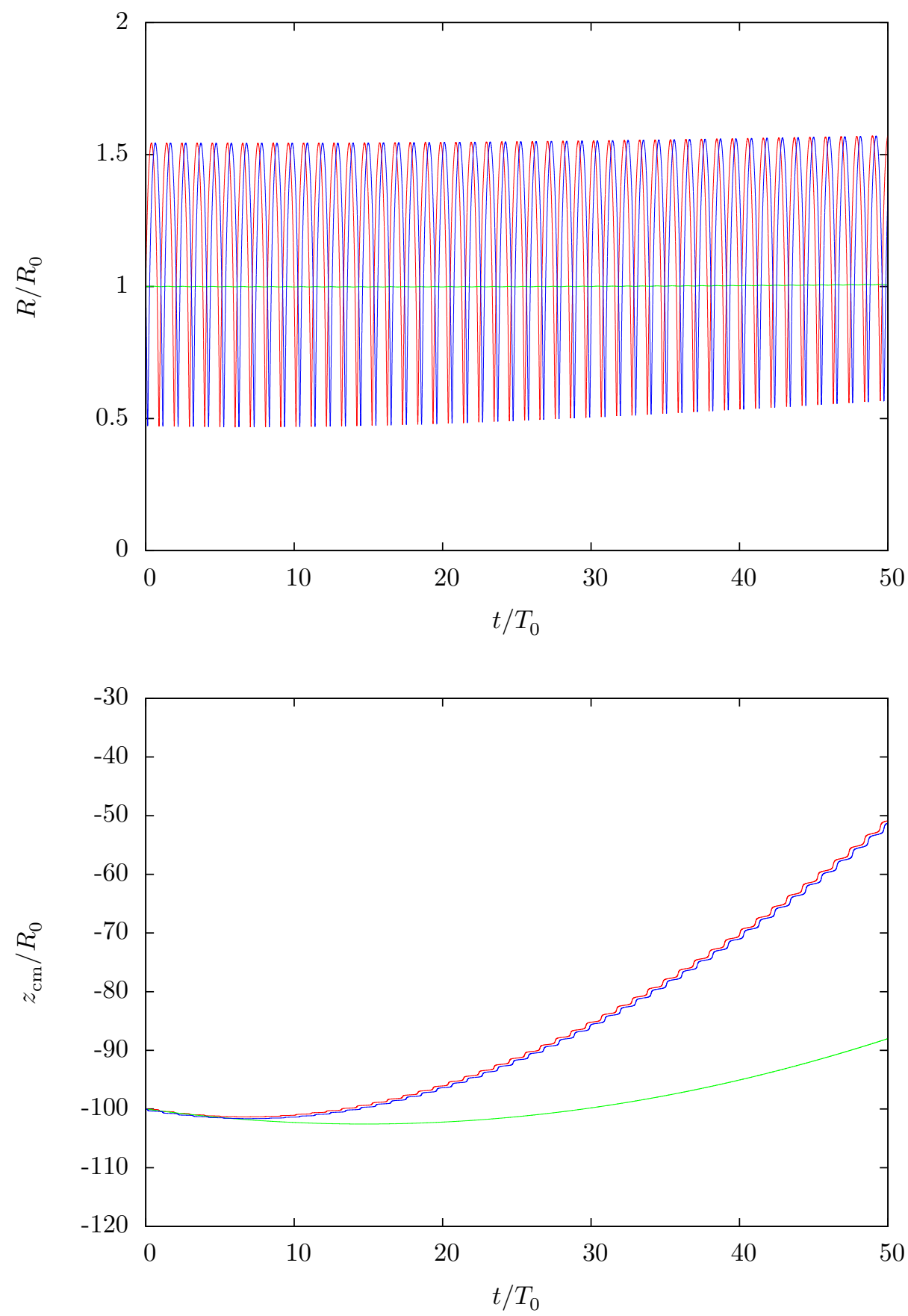

Figure 3. Bubble radius (top) and position of the center of mass (bottom). Data: $\dot{z}_{\mathrm{cm} 0}=$ $-1 \mathrm{~m} / \mathrm{s}$, and $\dot{R}_{0}=10 \mathrm{~m} / \mathrm{s}$ (red), $\dot{R}_{0}=0 \mathrm{~m} / \mathrm{s}$ (green), $\dot{R}_{0}=-10 \mathrm{~m} / \mathrm{s}$ (blue). 
E. De Bernardis, G. Riccardi
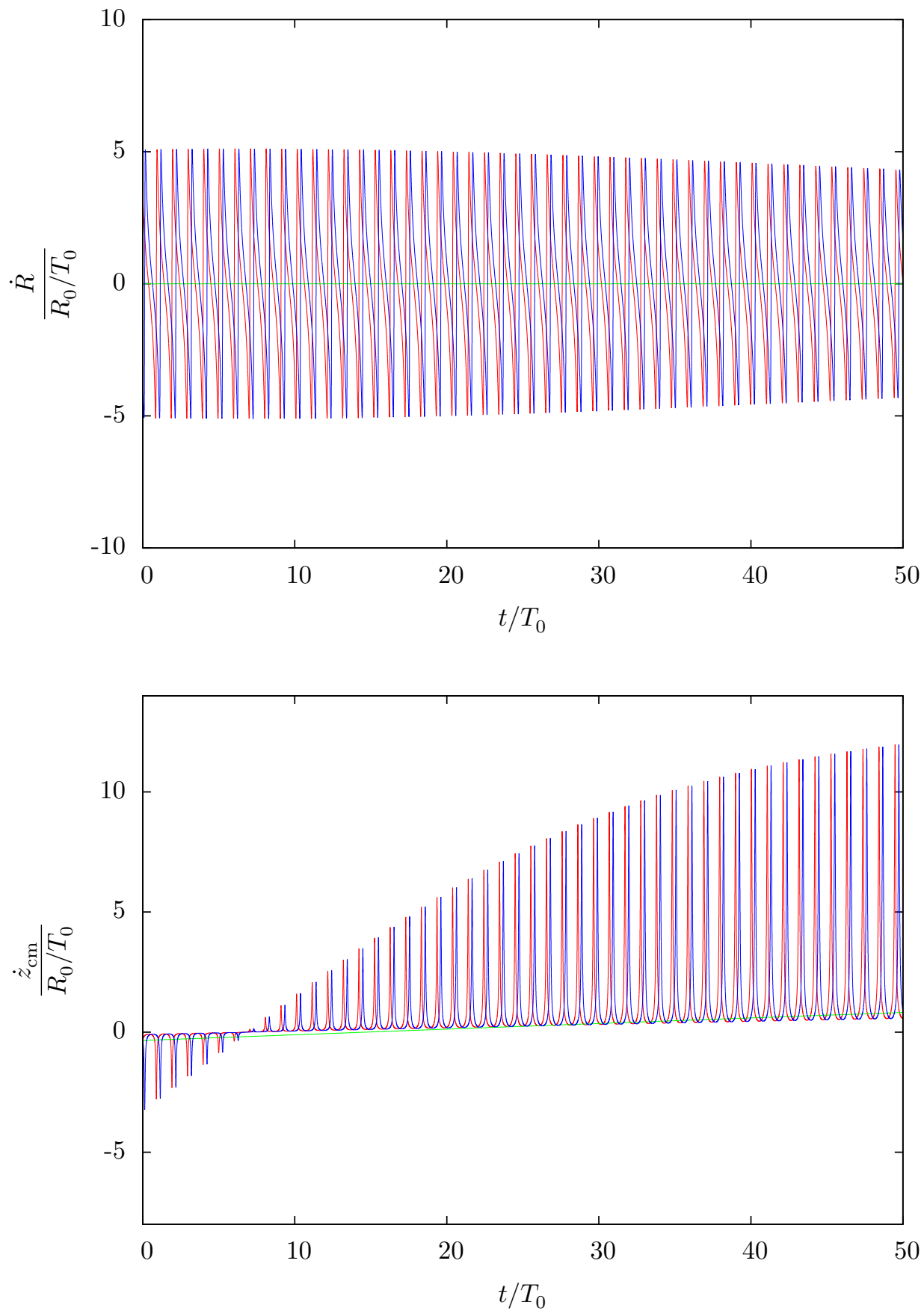

Figure 4. Radial velocity (top) and velocity of the center of mass (bottom). Data: $\dot{z}_{\text {cm0 }}=-1 \mathrm{~m} / \mathrm{s}$, and $\dot{R}_{0}=10 \mathrm{~m} / \mathrm{s}(\mathrm{red}), \dot{R}_{0}=0 \mathrm{~m} / \mathrm{s}$ (green), $\dot{R}_{0}=-10 \mathrm{~m} / \mathrm{s}$ (blue). 
Dynamics of a bubble rising in gravitational field
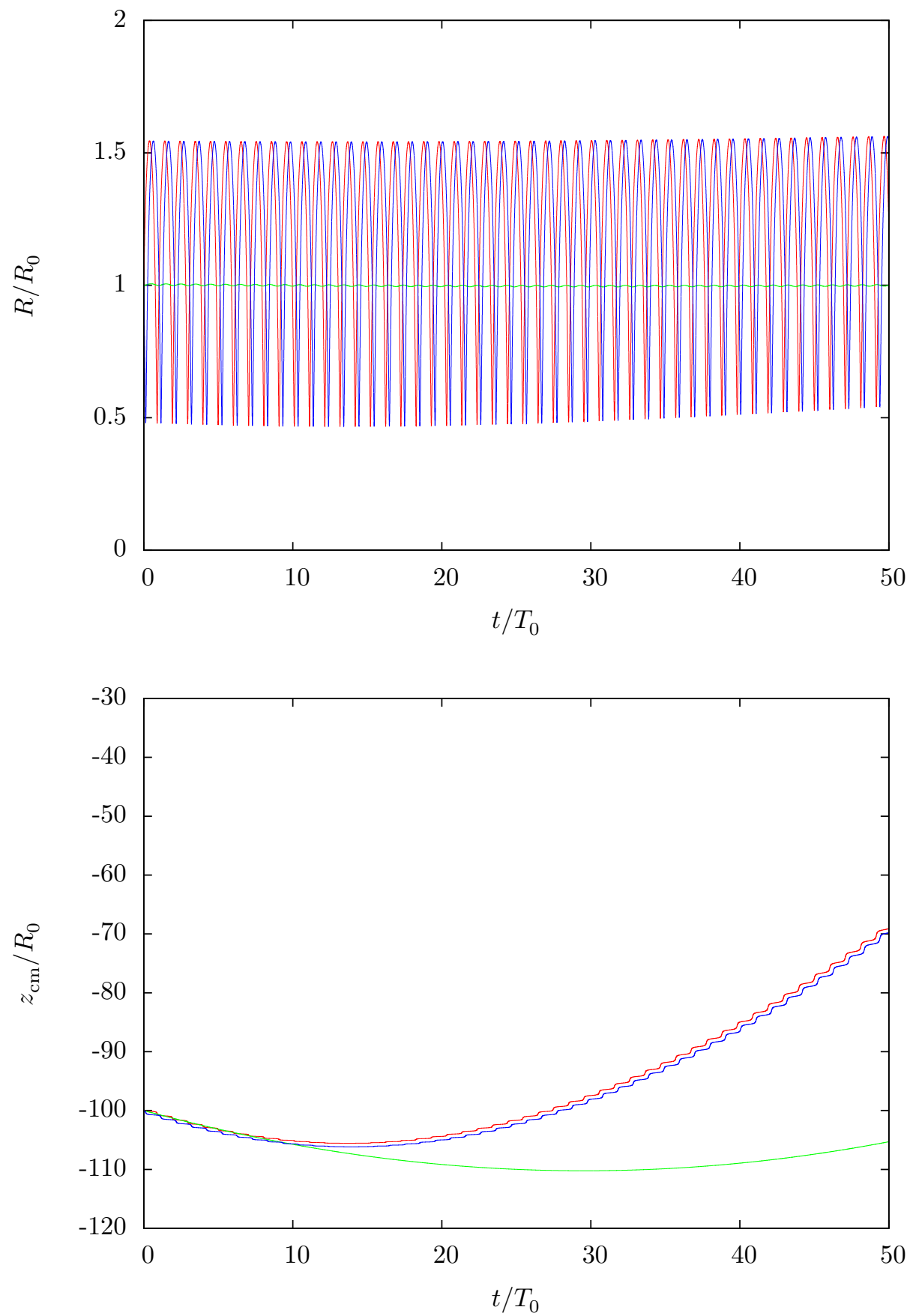

Figure 5. Bubble radius (top) and position of the center of mass (bottom). Data: $\dot{z}_{\mathrm{cm} 0}=$ $-2 \mathrm{~m} / \mathrm{s}$, and $\dot{R}_{0}=10 \mathrm{~m} / \mathrm{s}$ (red), $\dot{R}_{0}=0 \mathrm{~m} / \mathrm{s}$ (green), $\dot{R}_{0}=-10 \mathrm{~m} / \mathrm{s}$ (blue). 
E. De Bernardis, G. Riccardi
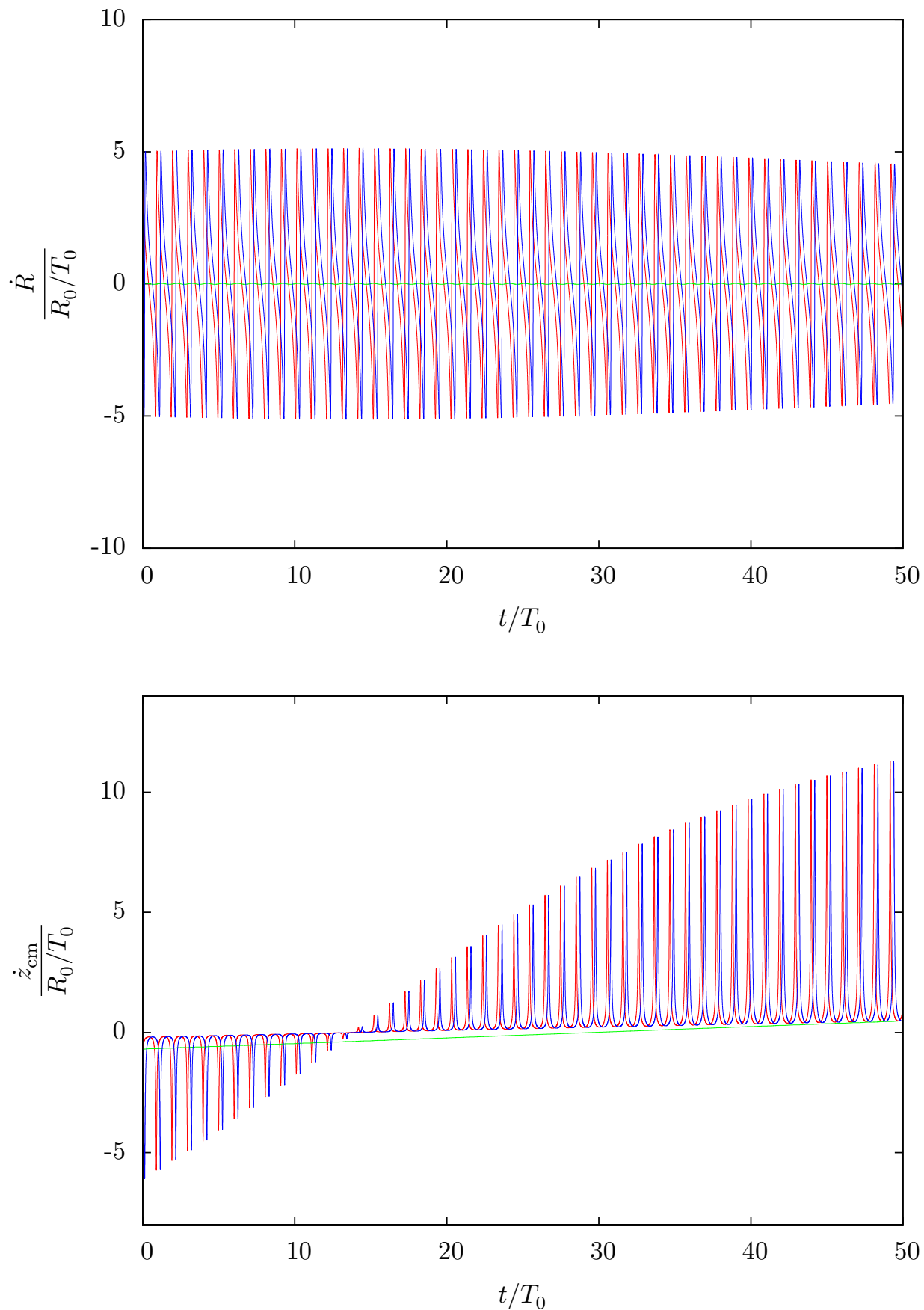

Figure 6. Radial velocity (top) and velocity of the center of mass (bottom). Data: $\dot{z}_{\mathrm{cm} 0}=-2 \mathrm{~m} / \mathrm{s}$, and $\dot{R}_{0}=10 \mathrm{~m} / \mathrm{s}\left(\right.$ red), $\dot{R}_{0}=0 \mathrm{~m} / \mathrm{s}$ (green), $\dot{R}_{0}=-10 \mathrm{~m} / \mathrm{s}$ (blue). 
Dynamics of a bubble rising in gravitational field
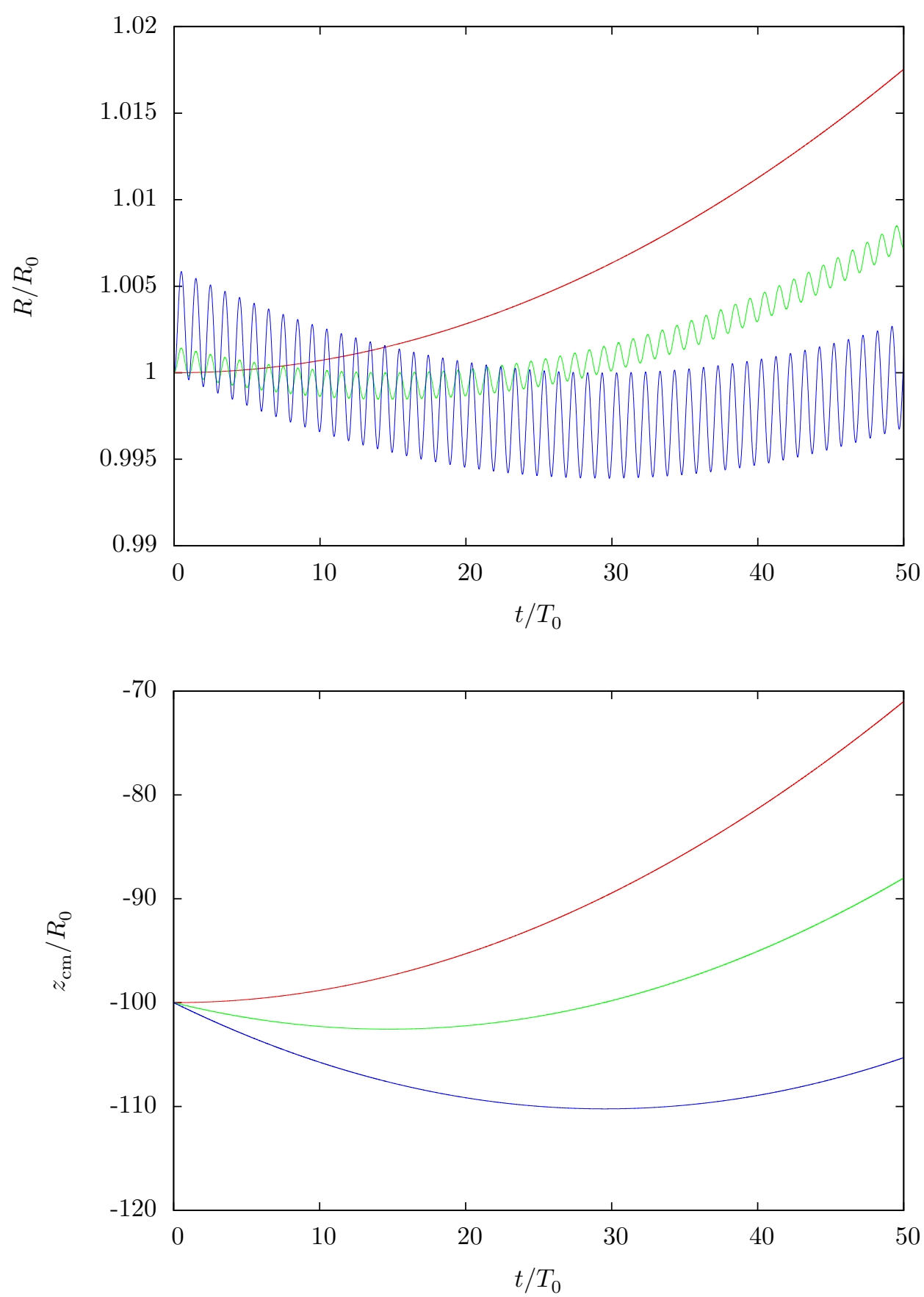

Figure 7. Bubble radius (top) and position of the center of mass (bottom). Data: $\dot{z}_{\mathrm{cm} 0}=$ $0 \mathrm{~m} / \mathrm{s}$ (red), $\dot{z}_{\mathrm{cm} 0}=-1 \mathrm{~m} / \mathrm{s}$ (green), $\dot{z}_{\mathrm{cm} 0}=-2 \mathrm{~m} / \mathrm{s}$ (blue). 


\section{E. De Bernardis, G. Riccardi}
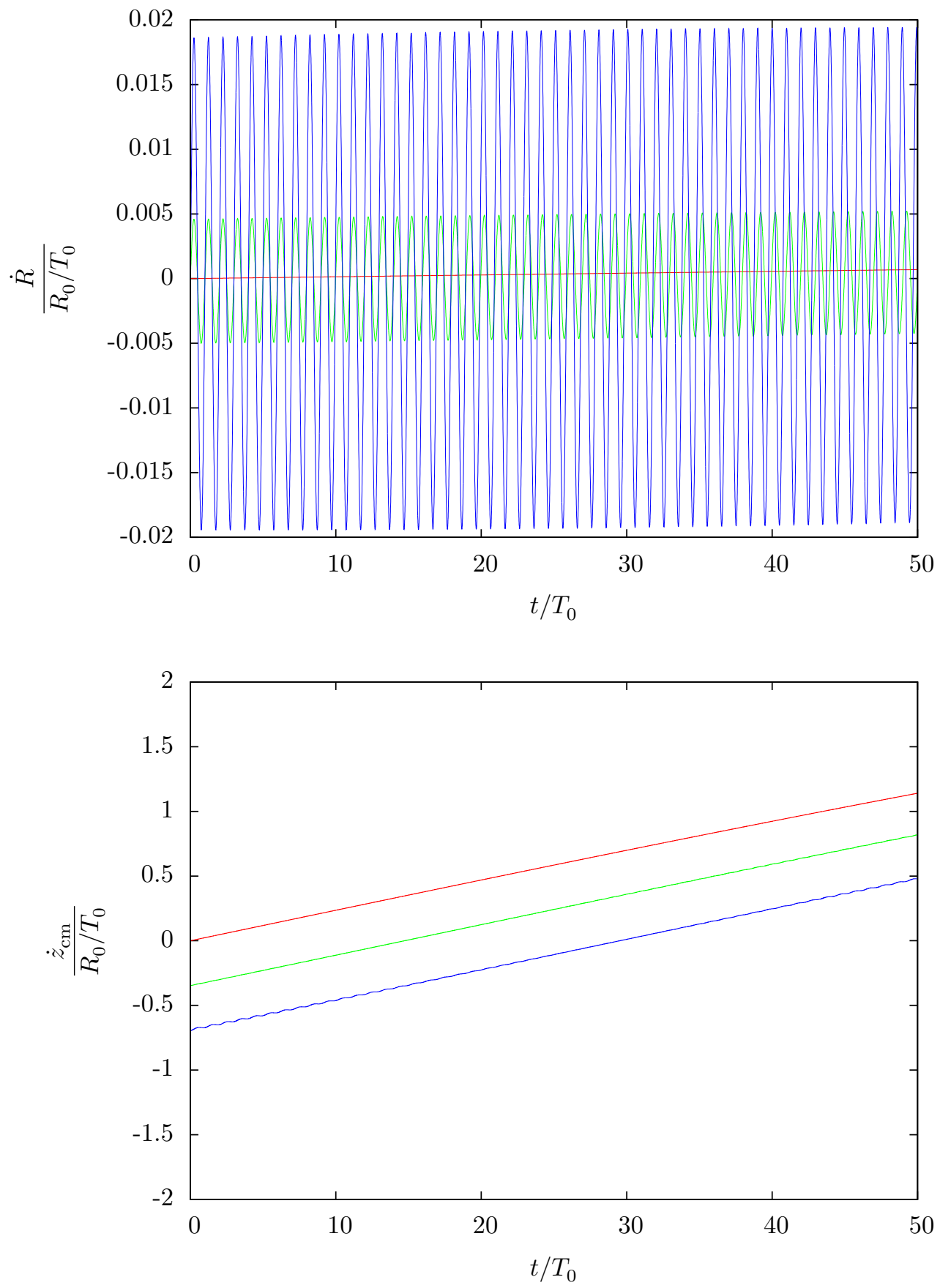

Figure 8. Radial velocity (top) and velocity of the center of mass (bottom). Data: $\dot{R}_{0}=$ $0 \mathrm{~m} / \mathrm{s}$, and $\dot{z}_{\mathrm{cm} 0}=0 \mathrm{~m} / \mathrm{s}$ (red), $\dot{z}_{\mathrm{cm} 0}=-1 \mathrm{~m} / \mathrm{s}$ (green), $\dot{z}_{\mathrm{cm} 0}=-2 \mathrm{~m} / \mathrm{s}$ (blue). 
Dynamics of a bubble rising in gravitational field
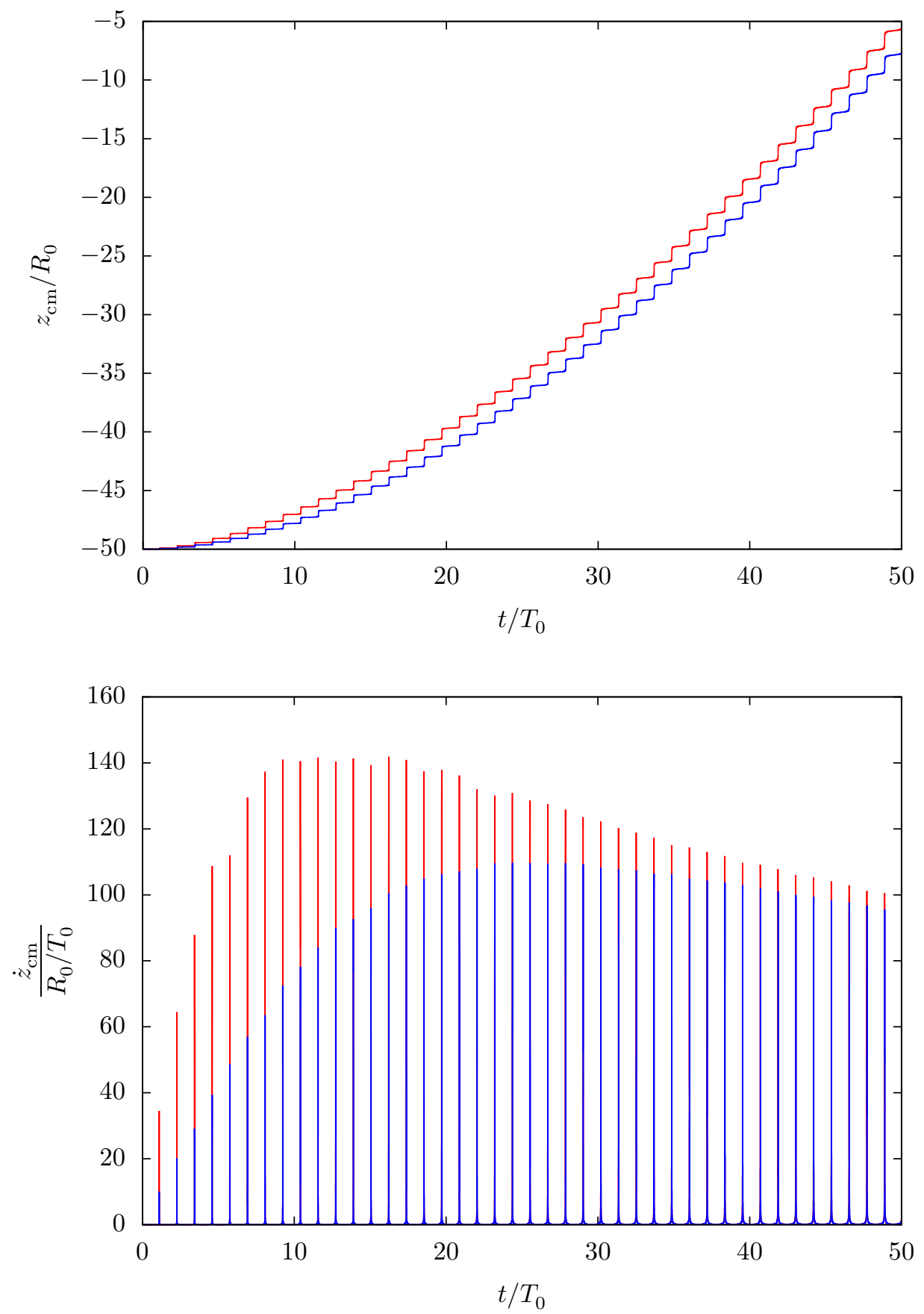

Figure 9. Position (top) and vertical velocity (bottom) of the center of mass. Data: $R_{0}=0.002 \mathrm{~m}, \dot{R}_{0}=20 \mathrm{~m} / \mathrm{s}, z_{\mathrm{cm} 0}=-0.1 \mathrm{~m}$, and $\dot{z}_{\mathrm{cm} 0}=0 \mathrm{~m} / \mathrm{s}$. Density ratio $\varepsilon$ calculated (blue) or enforced as $\varepsilon=0$ (red). 


\section{E. De Bernardis, G. Riccardi}

\section{Appendix. Modified Rayleigh-Plesset equation (13) derived from kinetic energy balance}

We use here the balance of kinetic energy to derive the equation for the evolution of the bubble radius. In presence of gravity, from Euler equations we obtain the equation of kinetic energy:

$$
\frac{D}{D t} \frac{|\boldsymbol{u}|^{2}}{2}=-\nabla \cdot\left(\boldsymbol{u} \frac{p+\rho g z-p_{\infty}}{\rho}\right)
$$

Note that $\boldsymbol{u} \sim 1 /|\boldsymbol{x}|^{2}$ and $\left(p+\rho g z-p_{\infty}\right) / \rho=-\partial \varphi / \partial t-|\boldsymbol{u}|^{2} / 2 \sim 1 /|\boldsymbol{x}|$ as $\boldsymbol{x} \rightarrow \infty$, so that the integral of $u_{n}\left(p+\rho g z-p_{\infty}\right) / \rho$ on the sphere at infinity vanishes. As a consequence, the balance of the kinetic energy $E$ of the liquid is

$$
\frac{d}{d t} \frac{E}{\rho}=\int_{\mathscr{B}} d S u_{n} \frac{p+\rho g z-p_{\infty}}{\rho} .
$$

The kinetic energy of the liquid is evaluated by using Equation (5):

$$
\frac{E}{\rho}=2 \pi \int_{R}^{\infty} d r r^{2} \int_{-1}^{1} d \mu \frac{|\boldsymbol{u}|^{2}}{2}=\frac{4 \pi}{3} R^{3}\left(\frac{3}{2} \dot{R}^{2}+\frac{1}{4} \dot{z}_{\mathrm{cm}}^{2}\right),
$$

while the integral in the right-hand side of Equation (14) is evaluated by using Equations (4), (11), and (12):

$$
\begin{aligned}
\int_{\mathscr{B}} d S u_{n} \frac{p+\rho g z-p_{\infty}}{\rho}= & 2 \pi R^{2} \int_{-1}^{1} d \mu\left(\dot{R} P_{0}+\dot{z}_{\mathrm{cm}} P_{1}\right) \times \\
& \times\left[P P_{0}+\left(\frac{3}{2} \dot{R} \dot{z}_{\mathrm{cm}}+\frac{1}{2} R \ddot{z}_{\mathrm{cm}}\right) P_{1}+\frac{3}{4} \dot{z}_{\mathrm{cm}}^{2} P_{2}\right] \\
& =4 \pi R^{2}\left(\dot{R} P+\frac{1}{2} \dot{R} \dot{z}_{\mathrm{cm}}^{2}+\frac{1}{12} R \frac{d}{d t} \dot{z}_{\mathrm{cm}}^{2}\right) .
\end{aligned}
$$

Once the Equations (15) and (16) are inserted into the balance (14), the equation for the evolution of the bubble radius (13) follows.

\section{Acknowledgements}

Activities described here were conducted under Grant of the Flagship Project RITMARE - The Italian Research for the Sea - coordinated by the National Research Council of Italy, funded by the Ministry of Education, University and Research within the National Research Programme 2011-2013. 


\section{Dynamics of a bubble rising in gravitational field}

\section{REFERENCES}

1. C. E. Brennen, Cavitation and Bubble Dynamics. New York: Oxford University Press, 1995.

2. A. J. Reddy and A. J. Szeri, Coupled dynamics of translation and collapse of acoustically driven microbubbles, J. Acoust. Soc. Am., vol. 112, no. 4, pp. 1346-1352, 2002.

3. A. J. Reddy and A. J. Szeri, Shape stability of unsteadily translating bubbles, Phys. Fluids, vol. 14, no. 7, pp. 2216-2224, 2002.

4. A. A. Doinikov, Translational motion of a spherical bubble in an acoustic standing wave of high intensity, Phys. Fluids, vol. 14, no. 4, pp. 1420 $1425,2002$.

5. A. A. Doinikov, Translational motion of a bubble undergoing shape oscillations, J. Fluid Mech., vol. 501, pp. 1-24, 2004.

6. A. A. Doinikov, Equations of coupled radial and translational motions of a bubble in a weakly compressible liquid, Phys. Fluids, vol. 17, no. $128101,2005$.

7. T. G. Leighton, The Acoustic Bubble. London: Academic Press, 1994.

8. S. J. Shaw, Translationand oscillation of a bubble under axisymmetric deformation, Phys. Fluids, vol. 18, no. 072104, 2006.

9. B. Yang, A. Prosperetti, and S. Takagi, The transient rise of a bubble subject to shape or volume changes, Phys. Fluids, vol. 15, no. 9, pp. 2640-2648, 2003.

10. E. Klaseboer and B. C. Khoo, A modified Rayleigh-Plesset model for a non-spherically symmetric oscillating bubble with applications to boundary integral methods, Eng. Anal. Bound. Elem., vol. 30, pp. 59$71,2006$.

11. L. Amaya-Bower and T. Lee, Single bubble rising dynamics for moderate Reynolds number using Lattice Boltzmann Method, Comput. Fluids, vol. 39, pp. 1191-2007, 2010.

12. D. Rodrigue, Generalized correlation for bubble motion, AIChE J., vol. 47, no. 1, pp. 39-44, 2001.

13. J. M. Gordillo, B. Lalanne, F. Risso, D. Legendre, and S. Tanguy, Unsteady rising of clean bubble in low viscosity liquid, Bubble Science, Engineering and Technology, vol. 4, no. 1, pp. 4-11, 2012.

14. G. S. Tuteja, D. Khattar, B. B. Chakraborty, and S. Bansal, Study of an expanding, spherical gas bubble in a liquid under gravity when the centre moves in a vertical plane, Int. J. Contemp. Math. Sciences, vol. 5, no. 22, pp. 1065-1075, 2010.

15. G. K. Batchelor, An Introduction to Fluid Dynamics. Cambridge: Cambridge University Press, 1967. 